to liver concentration of vitamin $\mathbf{A}$ in generally well-nourished surgical patients. Am J Clin Nutr 1984; 39: 898902.

7. Russell RM, Iber FL, Krasinki SD et al. Protein-energy malnutrition and liver dysfunction limit the usefulness of the relative dose response (RDR) test for predicting vitamin A deficiency. Hum Nutr Clin Nutr 1983; 37: 361-377.

8. Raghuramulu N, Underwood AB, Reddy V et al. Does relative dose response test re- flect vitamin A status? (Abstract) $X$ Meeting of IVACG, Session 2; 1985: Hyderabad, India, October 14-18.

9. Tanumihardjo SA, Olson JA. A modified relative dose-response assay employing 3,4dehydroretinol (vitamin $A_{2}$ ) in rats. $J$ Nutr 1988; 118: 598-603.

10. John A, Arunjyothi, Sivakumat B. Ammonium nitrogen to creatinine ratio in random urine samples as an index of vitamin A nutriture in children. Nutr Res 1988; 9: 153160.

\title{
PREVENTION OF FETAL GROWTH RETARDATION WITH LOW DOSE ASPRIN
}

Fetal growth retardation remains a substantial cause of fetal and neonatal mortality. It is related to chronic fetal hypo-perfusion, in most cases due to placental dysfunction. This is characterized by maladaptation of spiral arteries, endothelial injury and secondary thrombosis which leads to an imbalance of prostacyclin thrombaxene production. In the present study Uzan et al tested the efficacy of low-dose aspirin in preventing fetal growth retardation in a randomised, placebo-controlled, double-blind trial. 323 women at 15-18 weeks amenorrhoea were selected at twenty-five participating centres on the basis of fetal growth retardation and/or fetal death or abruptio placentae in at least one previous pregnancy. They were randomly allocated to groups receiving placebo, $150 \mathrm{mg} /$ day aspirin, or $150 \mathrm{mg} /$ day aspirin plus $225 \mathrm{mg} /$ day dipyridamole, for the remainder of the pregnancy. Mean birthweight was significantly higher in the treated than in the placebo group (2751 vs $2526 \mathrm{~g}$; difference $225 \mathrm{~g}$ ) and the frequency of fetal growth retardation in the placebo group was twice that in the treated group $(26 \%$ vs $13 \% ; p<0.02)$. The frequencies of stillbirth $(5 \%$ vs $1 \%)$ and abruptio placentae ( $8 \%$ vs $5 \%$ ) were also higher in the placebo than in the treated group. The benefits of aspirin treatment were greater in patients with two or more previous poor outcomes than in those with only one. In the second analysis, of aspirin only $(n=127)$ vs aspirin plus dipyridamole $(n=119)$, no significant differences were found. There was no excess of maternal or neonatal side-effects in the aspirin-treated patient. 